\title{
Fabrication and characterization of carbon based highly flexible smart battery fiber in the form of lithium ion battery
}

\author{
Amit Kumar Yadav ${ }^{1}$ and Kamal K Kar ${ }^{1,2, *}$ \\ ${ }^{1}$ Advanced Nanoengineering Materials Laboratory, Materials Science Programme, Indian Institute of \\ Technology Kanpur, Kanpur-208016, India \\ ${ }^{2}$ Advanced Nanoengineering Materials Laboratory, Department of Mechanical Engineering, Indian \\ Institute of Technology Kanpur, Kanpur-208016, India \\ *Email: kamalkk@iitk.ac.in
}

The objective of battery Fiber is to integrate power/energy applications with mechanical applications. In present study, carbon fiber (CF) possess high strength to weight ratio, also has large battery surface area is used as fibrous substrate. Based on the performance and compatibility, different materials have been chosen to act like current collector, cathode, electrolyte and anode layers. Generally, good conducting metals like silver and copper are chosen to act in form of current collector layer. Lithium Iron phosphate or Lithium cobalt oxide can be used for cathode. Poly ethylene oxide can be used as solid polymer electrolyte (SPE) and Lithium Titanium oxide can be used as anode. These materials are coated in the form of layers on the fiber substrate using various coating methods. Each fiber becomes a power source in itself.

The coating and optimization of cathode current collector layer (copper) and cathode layer (Lithium Iron Phosphate) and solid polymer electrolyte (Polyethylene oxide) and synthesis of anode material (Lithium titanium oxide) coating on the battery fibers (carbon substrate) has been reported. Multiple experimental methods have been adopted while doing so.

This involves "Cementation method [1] for coating copper on carbon fibers", plotting "V-I characteristic curves" to find the conductivities of copper coated and non-coated carbon fibers, preparation of Lithium Iron Phosphate as an active cathode material and "Electrophoresis Deposition [2]" for coating of Lithium Iron Phosphate on copper coated carbon fibers and "Dip coating method" for coating SPE and again EPD coating for anode layer deposition. Figure 1 shows electrophoretically deposited smooth cathode film and over cathode a transparent layer of PEO coating is coated. Optimization was done on each level using multiple Characterization techniques like "Scanning Electron Microscopy (SEM)", "X- RAY diffraction (XRD)", "Energy dispersive X-RAY spectroscopy (EDX)", etc. Finally, power fibers were studied for their battery characteristics (Figure 2). Their results have been studied and optimized.
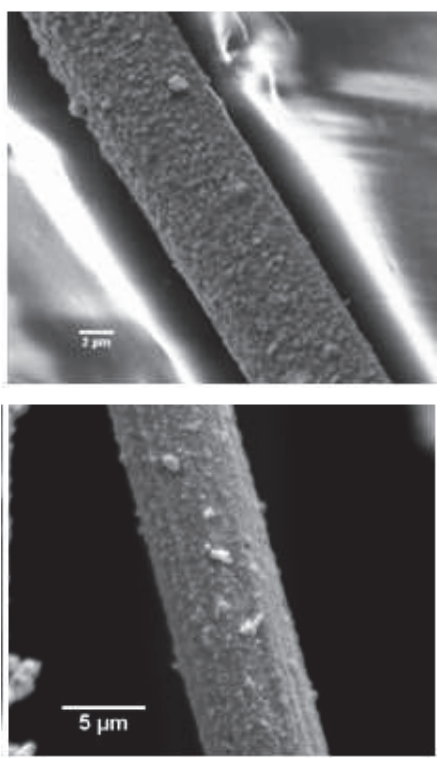

Figure 1: SEM images of $\mathrm{LiFePO}_{4}$ coated carbon fiber (CF) and $\mathrm{PEO}$ coating over $\mathrm{LiFePO}_{4}$ coated $\mathrm{CF}$

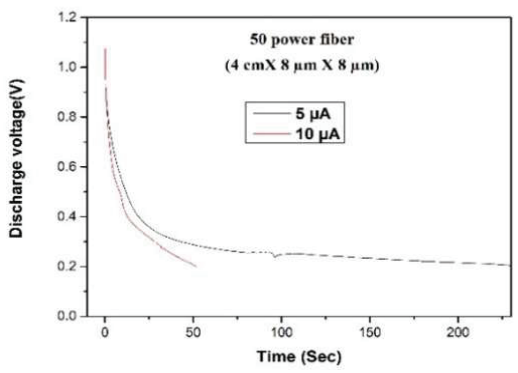

Figure 2: Discharge voltage as a function of discharge time with 5 and $10 \mu \mathrm{A}$ discharge current for around 50 power fibers

\section{References}

1. A.G. Kulkarni, B.C. Pai, N Balasubramaniam, J. Mater. Sci. 14(1979) 592598.

2. H. Mazor, D. Golodnitsky, L.Burstein, A.Gladkich, E.Peled, J. Power Sources 198(2012), 264-272. 